\title{
Efficient Soft Decoding of Raptor Codes for Satellite Broadcasting System
}

\author{
M. Zhang, S. Kim \\ Chonbuk National University \\ Korea
}

\begin{abstract}
In this paper, we propose an efficient soft iterative decoding method for raptor codes, which are customized for satellite broadcasting systems. The raptor codes presented in this paper are composed of a systematic LDPC code concatenated with another systematic Luby transform (LT) code. The outer codes are DVB-S2 and DVB-S2X defined forward error correction codes for interactive satellite broadcasting systems. For efficient soft decoding of the raptor codes, we represent both of the component codes with the same graph structure. Based on the Tanner graph drawn in terms of bit nodes and check nodes for each component code, we present an efficient joint iterative soft decoding method with a parallel process.
\end{abstract}

Keywords-rateless codes; raptor codes; satellite broadcasting; DVB-S2; iterative decoding

\section{INTRODUCTION}

Rateless codes, also known as fountain codes, were introduced to achieve capacity-achieving performance for the channel without requiring feedback or knowledge of the erasure conditions. Luby transform (LT) codes were the first class of rateless codes [1]. Raptor codes were designed as an extension of LT codes concatenating an LT code with a precode [2], and they are known to outperform LT codes on a wide variety of noisy channels [3]. A low-density parity check (LDPC) code is often used as the precode, and LDPC codes are famous for their capacity approximating performance due to soft iterative decoding [4].

Multimedia broadcast multicast services (MBMS) introduced in the third Generation partnership project (3GPP) uses forward error correction (FEC) scheme with rateless codes at the application layer to protect the video bit stream against packet loss [5]. The satellite system is an effective means to provide MBMS, and thus rateless codes are considered to provide satellite broadcasting services efficiently. For example, in order to overcome the heterogeneity and reduce the number of retransmissions, the packet level forward error correction (FEC) scheme using LT codes was incorporated in satellite data broadcasting system in [6]. Applications of raptor codes for the digital video broadcasting via satellite (DVB-S) system were considered in [7][8].

The channel conditions of wireless communication systems are usually much more severe, and attempts have been made to utilize soft decision information to improve the decoding performance [9]. Joint iterative soft decoding algorithms for raptor codes have been proposed that use the soft information produced by both component codes of raptor codes [10][11]. However, these joint decoding methods used multiple iterative loops that incurred a long decoding time. In this paper, we first propose an efficient parallel decoding algorithm for joint iterative decoding of raptor codes to reduce the decoding time. In the proposed decoding method, there is an initial decoding process of a component code, and the succeeding step invokes two component decoders simultaneously so that they can work in parallel. In this way, the proposed scheme does not require any additional memory or hardware, and reduces the decoding time by half.

Section 2 briefly reviews the encoding and decoding for raptor codes. Section 3 describes the basic concept of the proposed parallel decoding method, including a compact set of equations and an algorithm to execute the method. Section 4 presents performance simulation results, and section 5 concludes the paper.

\section{ENCODING AND DECODING OF CONCATENATED RATELESS CODES}

We consider raptor codes that are composed of outer LDPC codes concatenated with inner LT codes. They are encoded first using the information I, of length k', to u, of length $\mathrm{k}$, by applying the outer LDPC code using a parity check matrix H of the LDPC code; then, the codeword $c$ is generated by the inner LT encoder with a predetermined degree distribution $\rho$ [1][2].

In the decoding process, whether it is carried out in a tandem or joint manner [10][11], LT decoding should be carried out first so that its soft output information can be provided to the subsequent LDPC decoding process. If joint decoding is performed, then soft output information from the LDPC decoding is fed back to the LT decoder, and this process is iterated through the exchange of soft information. The soft information used during the iterative decoding process is generally in the form of log-likelihood ratios (LLRs), and exchanges of these LLRs are made through the Tanner graphs of both component codes [11], as shown in Fig. 1.

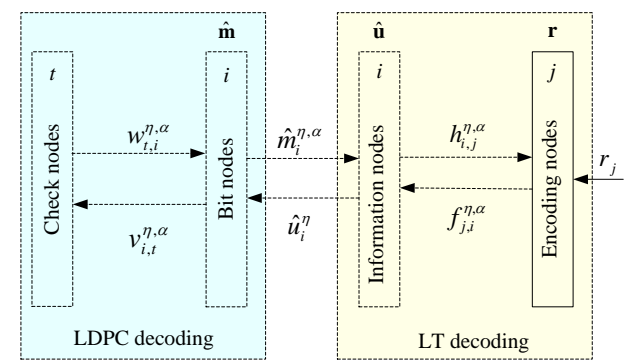

FIGURE I. INFORMATION FLOW THROUGH THE TANNER GRAPH FOR SOFT ITERATIVE DECODING OF RAPTOR CODES

Referring to the soft information flow through the Tanner graph in Fig. 1, in conventional soft tandem decoding, the LT decoder takes the demodulated soft information $r$, of length $\mathrm{n}$, as a priori information on the 
encoding nodes, and produces soft information $\hat{\mathbf{u}}$, of length $\mathrm{k}$, on the information nodes by means of iterative exchange of soft LLRs between the information and encoding nodes.

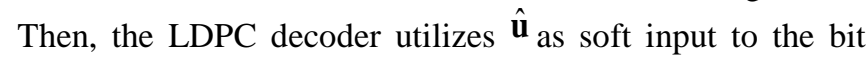
nodes, and estimates a soft output $\hat{\mathbf{m}}$ by iterative exchange of soft LLRs between the bit nodes and the check nodes. For joint iterative decoding, the hard decision on $\hat{\mathbf{m}}, \hat{\mathbf{c}}$, is used to evaluate the parity check equation, $\hat{\mathbf{c}} \mathbf{H}^{T}=0$. If the parity check equation is not satisfied, $\hat{\mathbf{m}}$ from the LDPC decoder is passed back to the LT decoder and the decoding is repeated.

\section{PROPOSED PARALlEL SOFT DECODING FOR RAPTOR CODES}

Conventional joint iterative decoding process involves three iterative loops, including iterative loops inside LT and LDPC decoders, and iterative loops between two decoders [10][11]. We note that, after an initial iteration, not only the LT decoder but also LDPC decoder can be activated. In this case, the LT decoder and the LDPC decoder can start the decoding by using extrinsic information provided by the other decoder. By this way, two decoders can be processed in parallel, and neither additional hardware processors nor memories are required.

With this basic concept, the proposed parallel decoding method is explained as follows. Referring to Fig. 1, first, soft input vector for to the LT decoder, $\hat{\mathbf{r}}$, is estimated by using a soft demodulator such as in [12]. Then, soft iterative decoding with either a sum-product or min-sum algorithm is performed by alternatively estimating LLRs between the encoding node, $\mathrm{j}$, and information node, $\mathrm{i}$, where $1 \leq \mathrm{j} \leq \mathrm{n}$ and $1 \leq \mathrm{i} \leq \mathrm{k} . \quad f_{j, i}^{\eta, \alpha}$ is the LLR estimated from encoding node $\mathrm{j}$ to information node $\mathrm{i}$, while $h_{i, j}^{\eta, \alpha}$ is the one from information node $\mathrm{i}$ to encoding node $\mathrm{j}$, where $\eta$ and $\alpha$ are the indices indicating the number of iterations for the joint and LT decoding, respectively.

The decoding process is first started by initialization of $h_{i, j}^{\eta, \alpha}=0$, with $\eta=\alpha=0$. With these initial values, the LT decoder updates the LLR values between the encoding and information nodes alternatively using the following equations, assuming the sum-product algorithm is used [9]:

$$
f_{j, i}^{\eta, \alpha}=2 \tanh ^{-1}\left(\tanh \frac{r_{j}}{2} \cdot \prod_{x \in N_{j}, x \neq i} \tanh \frac{h_{x, j}^{\eta, \alpha-1}}{2}\right),
$$

where $\mathrm{Nj}$ is the index set of the information nodes connected with encoding node, $\mathrm{j}$.

$$
h_{i, j}^{\eta, \alpha}=\sum_{e \in \varepsilon_{i}, e \neq j} f_{e, i}^{\eta, \alpha}
$$

where $\varepsilon_{i}$ is the index set of the encoding nodes connected with information node $i$. This iterative process is continued until we reach the maximum iteration number, $\alpha_{\max 0}$. Finally, the soft output information is calculated by:

$$
\hat{u}_{i}^{\eta}=\sum_{e \in \varepsilon_{i}} f_{e, i}^{\eta, \alpha}
$$

Afterward, LDPC decoder is activated using the following initialization:

$$
v_{i, t}^{\eta, 0}=\hat{u}_{i}^{\eta-1}, t \in R_{i},
$$

where $v_{i, t}^{\eta, \alpha}$ is the LLR estimated from bit node $\mathrm{i}$ to check node $\mathrm{j}$, and $\mathrm{Ri}$ is the index set of the check nodes connected with bit node i. Afterwards, the LDPC decoder alternatively estimates the following LLR values:

$$
\begin{aligned}
w_{t, i}^{\eta, \alpha} & =2 \tanh ^{-1}\left(\prod_{s \in C_{t}, s \neq i} \tanh \frac{v_{s, t}^{\eta, \alpha-1}}{2}\right), \\
v_{i, t}^{\eta, \alpha} & =\sum_{y \in R_{i}, y \neq t} w_{y, i}^{\eta, \alpha},
\end{aligned}
$$

where here $w_{t, i}^{\eta, \alpha}$ is the LLR estimated from the check node $t$ to bit node $i$, and $C_{t}$ is the index set of the bit nodes connected with check node $t$, where $1 \leq t \leq k-k^{\prime}$. Subsequently, the soft output on bit nodes is estimated by:

$$
\hat{m}_{i}^{\eta, \alpha}=\sum_{t \in R_{i}}\left(w_{t, i}^{\eta, \alpha}+v_{i, t}^{\eta, \alpha}\right) .
$$

If the parity check equation for the LDPC code is satisfied, the decoding process will be terminated. Otherwise, this iterative process is continued until we reach the maximum iteration number, $\alpha_{\max 1}$. Afterwards, LT decoder continues its iterative process with a new initialization as follows:

$$
h_{i, j}^{\eta, 0}=h_{i, j}^{\eta-1, \alpha}+\hat{m}_{i}^{\eta-1, \alpha}, j \in \varepsilon_{i}
$$

where $\alpha=\alpha_{\max 1}$. At the same time LDPC decoder is performed in parallel with the initialization as in (4). Using these initialized values, the LT decoder estimates LLR values in (1) and (2) alternatively, in parallel with the LDPC decoder estimate alternative LLR values in (5) and (6).

Both of the LT and LDPC decoders will repeat the above process in parallel until they reach the maximum number of inner iterations, $\alpha=\alpha_{\max 1}$. After exiting the inner loop, both decoders exchange their soft outputs utilizing the initialization process defined in (4) and (8), and this will be continued until they reach the maximum number of outer iterations, Fig. 3 shows how the extrinsic information is exchanged during the parallel decoding process, and the number with parenthesis in each box represents the equation number to estimate LLRs. 


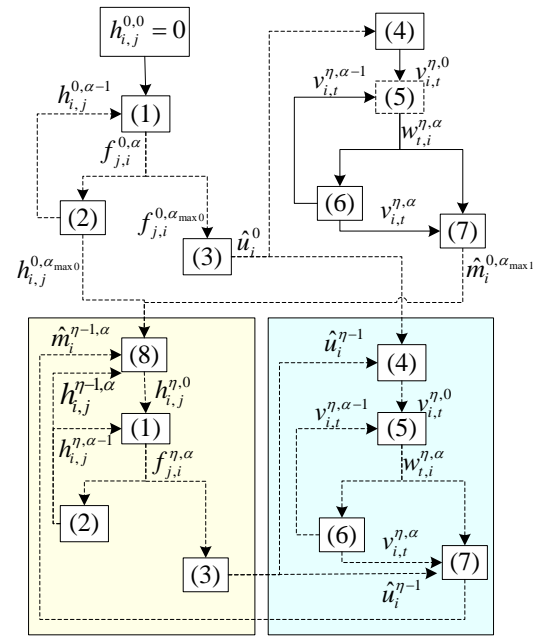

FIGURE II. EXCHANGE OF SOFT INFORMATION IN THE PROPOSED PARALLEL DECODING METHOD

\section{Simulation Results}

We simulated the performance of the proposed parallel soft iterative decoding algorithm on an additive white Gaussian noise (AWGN) channel with binary phase shift keying (BPSK) modulation scheme. We used systematic raptor codes. As the inner and outer codes, LT codes with a degree distribution specified in [13] and LDPC code with the normal frame size and code rate of $1 / 4$ specified in DVB-S2 standard were used [14][15].

Fig. 3 shows the BER performance comparison when $\left(\eta_{\max }, \alpha_{\max 0}, \alpha_{\max 1}\right)=(50,1,1)$, where $R=k / n$ is the code rate of the raptor code. We note that the proposed parallel decoding method can reduce the decoding time by half due to the concurrent activation of both component decoders. The BER simulation result in Fig. 3 shows that the proposed parallel decoding method produces almost the same performance as the conventional sequential decoding method with twice faster decoding speed.

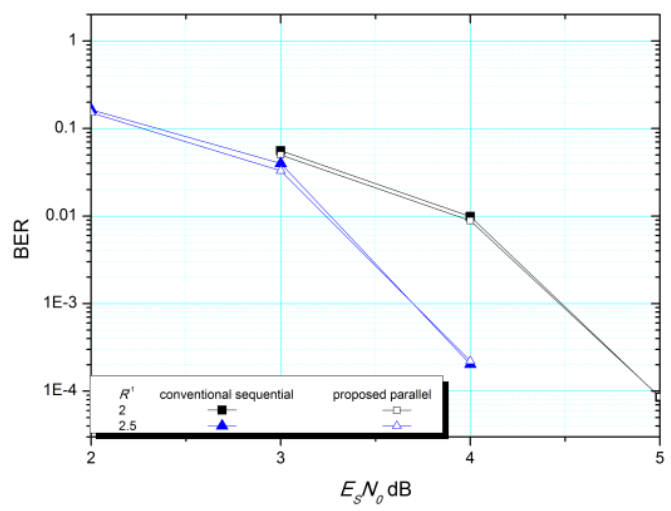

FIGURE III. BER PERFORMANCE COMPARISON WITH DIFFERENT R-1 VALUES WHEN (HMAX, AMAX0, AMAX1) $=(50,1$, 1)

\section{CONCLUSIONS}

In this paper, we presented an efficient soft iterative decoding of raptor codes for satellite broadcasting systems. The simulation results demonstrated that the proposed parallel iterative decoding scheme produces approximating performance as the conventional serial decoding scheme with nearly twice faster speed.

\section{REFERENCES}

[1] M. Luby, LT Codes, Proc. of 43rd Annual IEEE Symp. on the Found. of Comp. Sci, pp. 271-280, 2002.

[2] A. Shokrollahi, Raptor Codes, IEEE Transaction on Information Theory, 52(6), pp. 2551-2567, 2006.

[3] R. Palanki and J. Yedidia, Rateless codes on noisy channel, Proc. of ISIT, pp.38, 2004.

[4] R. Gallager, "Low-density parity check codes," IRE Trans. Information Theory, pp. 21-28, 1962.

[5] R. Hamzaoui, S.Ahmad, and M. AL-Akaidi, Reliable Wireless Video Streaming with Digital Fountain Codes, pp. 1-17.

[6] H. Zhu, G. Li, and Z. Xie, Advanced LT codes in Satellite Data Broadcasting System, Proc. of 11th IEEE Singapore International Conference on Communication Systems, pp. 1430-1435, Nov. 2008.

[7] P. Cateldi, M. Gerla, and F. Zampognaro, Rateless Codes for File Transfer over DVB-S, Proc. of First International Conference on Advances in Satellite and Space Communications, pp. 7-12, Jul. 2009.

[8] M. Hagh and M. Soleymani, Constellation Rotation for DVB Multiple Access Channels with Raptor coding, IEEE Transactions on Broadcasting, 59(2), pp. 290-297, Jun. 2013.

[9] H. Jenka, T. Mayer, T. Stockhammer, and W. Xu, Soft Decoding of LT-codes for Wireless Broadcast, Proc. of IST Mobile, Dresden, Germany, 2005.

[10] A. Venkiah, C. Poulliat, and D. Declercq, Analysis and Design of Raptor Codes for Joint Decoding using Information Content evolution, Proc. of IEEE ISIT 2007, pp. 421-425, 2007.

[11] M. Zhang, S. Kim, and X. Jiang, Joint Iterative Soft Decoding for Raptor Codes, Proc. of ISCE 2013, Hsinchu, Taiwan, pp. 25-26, 2013.

[12] M. Zhang, S. Kim, J. Lee, S. Song, W. Kim, and Y. Cho, Universal Soft Decision Demodulator for M-ary Adaptive Modulation Systems, Proceedings of APCC 2012, Jeju, Korea, pp. 574-578, 2012.

[13] K. Hu, J. Castura, and Y. Mao, Performance-Complexity Tradeoffs of Raptor Codes over Gaussian Channels, IEEE Communication Letters, 11(4), pp. 343-345, 2007.

[14] ESTI EN 302307 V1.3.1: Second Generation Framing Structure Channel Coding and Modulation System for Broadcasting Interactive Service, News Gathering and Other Broadband Satellite Applications (DVB-S2), 2013.

[15] DVB Document A83-2, Digital Video Broadcasting (DVB); Second generation framing structure, channel coding and modulation systems for Broadcasting, Interactive Services, News Gathering and other broadband satellite applications, Part II: S2-Extensions (DVB-S2X) (Optional), March 2014. 